\title{
Successful Treatment of Ulcerous Skin Lesion Caused by Mistaken Intradermal Injection of BCG (Bacille de Calmette et Guerin) Vaccine with Anti-tuberculous Drugs and Systemic Administration of Corticosteroid
}

\author{
Koji Watanabe, Katsuji Teruya, Yoshimi Kikuchi and Shinichi Oka
}

\begin{abstract}
"Tokyo 172" BCG vaccine designed for percutaneous inoculation is widely used in Japan. This vaccine is a much higher concentration of $M$. bovis BCG than that of the BCG vaccine designed for intradermal inoculation widely used in other countries. A patient who suffered from an ulcerous skin lesion as a result of the intradermal injection of "Tokyo 172" BCG vaccine accidentally was referred to our hospital. Systemic corticosteroid was administered with anti-tuberculous drugs, achieving a complete improvement of the skin lesion.
\end{abstract}

Key words: intradermal injection, BCG, corticosteroid, anti-tuberculous drug, skin lesion

(Inter Med 48: 1323-1325, 2009)

(DOI: 10.2169/internalmedicine.48.2195)

\section{Introduction}

Tuberculin skin test (TST) is widely performed for school age children or upon employment in Japan. Cases have been reported by media where BCG vaccine rather than Purified Protein Derivative, has been mistakenly injected intradermally for TST, causing a severe ulcerous skin lesion and leaving a prominent scar. However, medical articles of such treatment have been rarely reported (7), therefore, there are few treatment options available for the best means to prevent severe sequelae. We report one case which was referred to our hospital after accidental intradermal injection of BCG vaccine.

\section{Case Report}

A 24-year-old woman pharmacist received a 2-step tuberculin skin test (TST) upon employment at a clinic. The first TST was performed on her first visit of our hospital, and a reaction (redness diameter $7 \times 10 \mathrm{~mm}$ ) of redness without induration developed.
After two weeks the second TST was performed on her right arm, she noticed a blistering lesion at the injection site and severe pain from that night. The following day, the clinic checked the vial administered to her, and it was revealed that BCG vaccine (Tokyo 172), rather than Purified Protein Derivative for TST, had been accidentally inoculated intradermally. The patient was referred to our hospital. On her first visit, she was afebrile and her general condition was good. No surface lymph nodes were palpable. Her right arm was remarkably swollen and enlarged with a blistering lesion at the BCG injection site surrounded by a diameter of $200 \times 120 \mathrm{~mm}$ of skin redness (Fig. 1). No abnormal data but mild elevation in CRP was seen in complete blood cell counts and serum chemistry (Table 1). Acid-fast bacilli were detected with Ziehl-Nielsen staining of the specimen obtained from the blister puncture, and it was later confirmed to be Mycobacterium bovis BCG in the culture result which is susceptible to all of the anti-tuberculous drugs. Treatment with oral prednisolone $40 \mathrm{mg} / \mathrm{day}(1 \mathrm{mg} / \mathrm{kg} / \mathrm{day})$ and antituburculous drugs including isoniazid, rifampicin, and ethambutol were initiated from 16th day. Oral prednisolone was discontinued on the 7 th day of treatment after confirm- 
ing the significant improvement of the symptoms in the right arm (Fig. 2). Oral prednisolone (40 mg/day), however, was restarted on the 21 st day of treatment after the inflammatory changes in the right arm had started to worsen again (Fig. 3). Afterwards, the dosage of prednisolone was gradually reduced guided by the careful observation of local findings in the right arm, and was discontinued on the 63th day of treatment (Table 2). On the day of prednisolone withdrawal, an ulcerous lesion without any pain was noted in the arm (Fig. 4). After 24 weeks the anti-tuburculous treatment was completed, when the lesion was completely cured with-

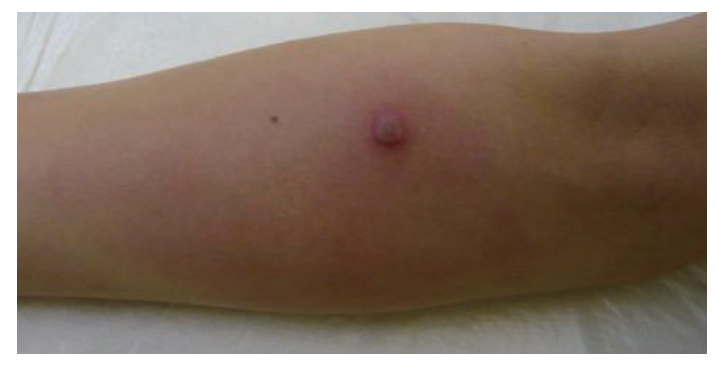

Figure 1. Blistering lesion surrounded by a redness area with much pain was seen at BCG-injection site on her first visit of our hospital.

Table 1. Laboratory Data on Her First Visit of Our Hospital

\begin{tabular}{|c|c|c|c|}
\hline WBC & $6.11 \times 10 \mathrm{E} 3 / \mu \mathrm{L}$ & Alb & $4.5 \mathrm{~g} / \mathrm{dL}$ \\
\hline neutro & $74.8 \%$ & T-bil & $1.0 \mathrm{mg} / \mathrm{dL}$ \\
\hline lymph & $17.3 \%$ & AST & $15 \mathrm{IU} / \mathrm{L}$ \\
\hline mono & $3.3 \%$ & ALT & $16 \mathrm{IU} / \mathrm{L}$ \\
\hline eosino & $4.4 \%$ & LDH & $147 \mathrm{IU} / \mathrm{L}$ \\
\hline baso & $0.2 \%$ & ALP & $155 \mathrm{IU} / \mathrm{L}$ \\
\hline RBC & $4.34 \times 10 \mathrm{E} 6 / \mu \mathrm{L}$ & $\begin{array}{c}\text { ChE } \\
\gamma-\text { GTP }\end{array}$ & $\begin{array}{r}325 \mathrm{IU} / \mathrm{L} \\
15 \mathrm{IU} / \mathrm{L}\end{array}$ \\
\hline $\mathrm{Hgb}$ & $13.6 \mathrm{~g} / \mathrm{dL}$ & \multirow{2}{*}{\multicolumn{2}{|c|}{$\begin{array}{l}\text { Cre } 0.55 \mathrm{mg} / \mathrm{dL} \\
\text { CRP } 0.93 \mathrm{mg} / \mathrm{dL}\end{array}$}} \\
\hline Hct & $39.9 \%$ & & \\
\hline
\end{tabular}

out any scar (Fig. 5).

\section{Discussion}

"Tokyo 172" BCG vaccine is designed to be used for percutaneous inoculation; it contains $3 \times 10^{9}$ colony forming units/mL, a 160-fold higher concentration of M. bovis BCG than other BCG vaccines inoculated intracutaneously $(1,2)$. Intradermal injection of "Tokyo 172" BCG to a person with a positive TST result could lead to serious complications such as intractable ulcer formation, because an excessive im-

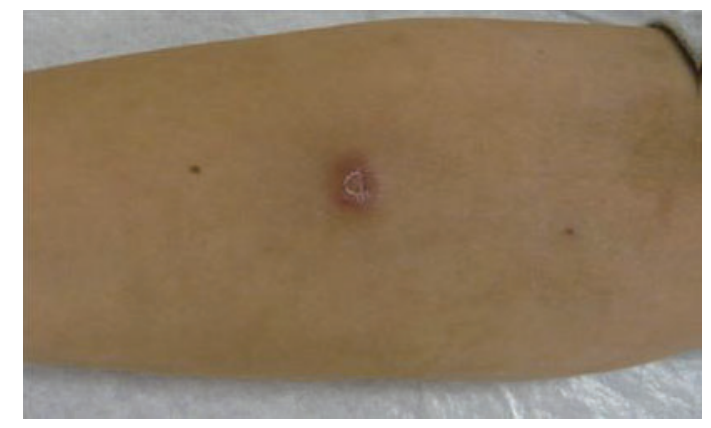

Figure 2. Significant improvement of skin lesion was seen after 1 week of corticosteroid therapy.

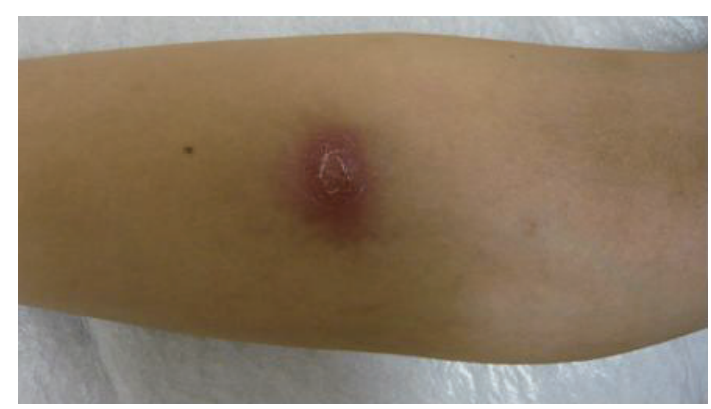

Figure 3. Local lesion of injection site was worsened again 21st day of treatment. So we started corticosteroid therapy again.

Table 2. Clinical Course of Our Hospital

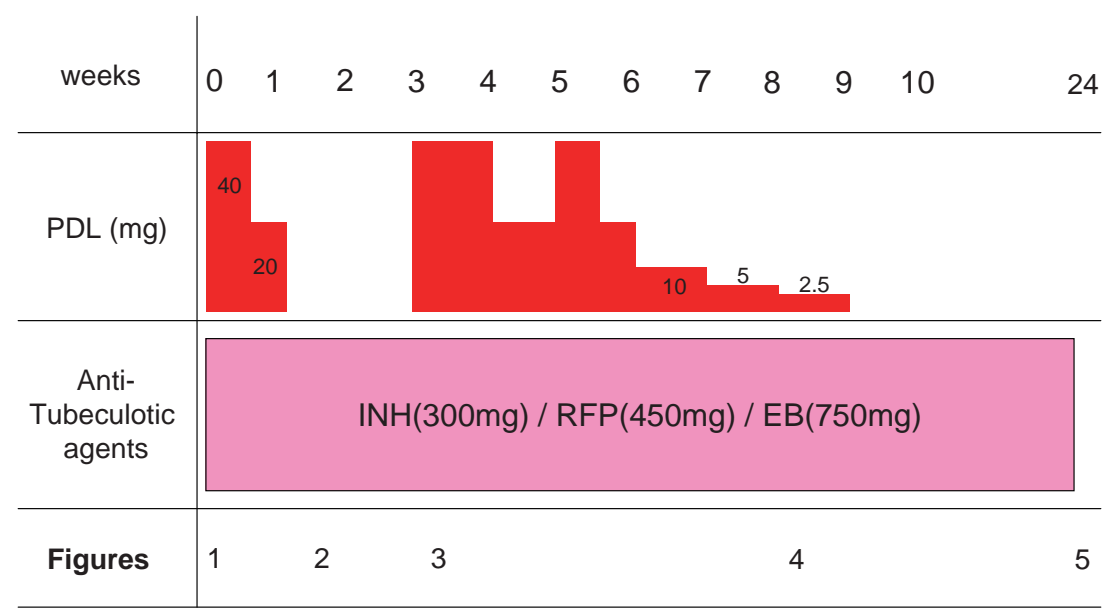




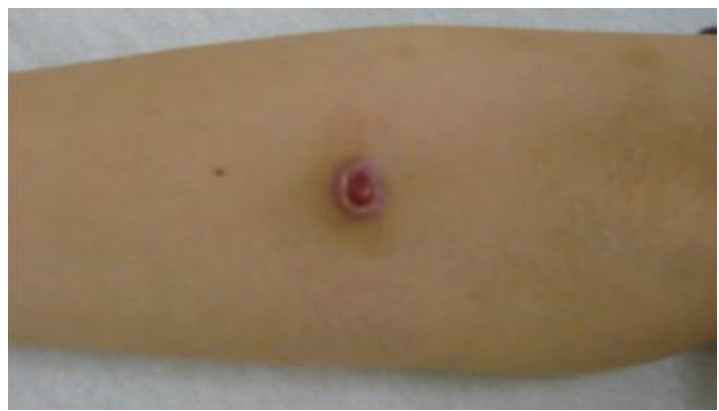

Figure 4. Skin lesion was improved when corticosteroid was tapered off at 64th day of treatment.

mune response could be induced by the large quantity of antigen stimulation. Mycobacterium bovis Tokyo strain is susceptible to isoniazid, rifampicin, ethambutol and streptomycin, but resistant to pyrazinamide in vitro (3). BCG-related diseases after vaccination or BCG bladder instillation have been treated with anti-tuberculous drugs in reported cases (4-6). In a few cases after BCG bladder instillation, corticosteroid was successfully administered with antituberculous drugs, but it is still controversial.

We found only one case report describing the clinical course after mistaken intradermal injection of "Tokyo 172" BCG (7). In that case, the patient had oral anti-tuberculous drugs containing isoniazid and rifampicin for 6 months coadministered with topical rifampicin ointment. Corticosteroid was not included in the treatment. After 2 years follow up, a severe scar was left on his arm, and cosmetic surgery was

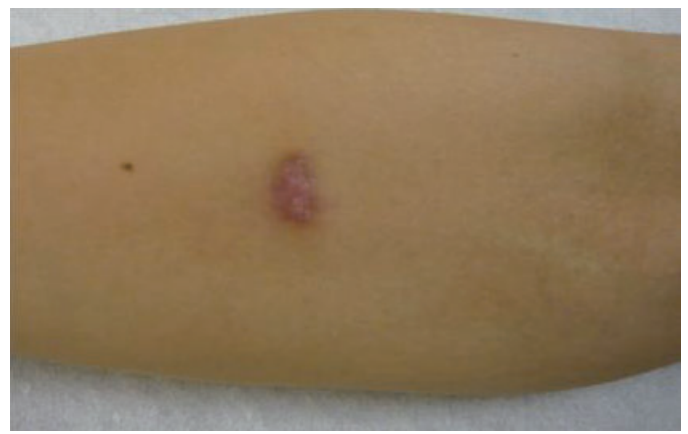

Figure 5. A tiny scur was left at injection site after completion of 6 month antituberculous treatment.

under consideration at that time. In the present case, the patient already had very strong inflammation accompanied with a blistering changes at the BCG injection site on the first visit day, suggesting that excess immune reaction was triggered after large quantity of antigen exposure to a already-immune host. So suppression of excess inflammation as well as treatment against M. bovis BCG was necessary. Treatment with anti-tuburculous drugs and oral steroid successfully suppressed the local inflammation and led to complete cure without any skin scarring formation. Because only a few reports were available about the management of cases of accidental injection of BCG and this is the first case which received corticosteroid, the case report is of great value suggesting that systemic steroid might have a favorable effect for a better outcome of such a local lesion.

\section{References}

1. Hawkridge A, Hatherrill M, Little F, et al. Efficacy of percutaneous versus intradermal BCG in the prevention of tuberculosis in South African infants: randomized trial. B M J 337: a2052, 2008.

2. Yamamoto S, Yamamoto T. Historical Review of BCG Vaccine in Japan. Jpn J Infect Dis 60: 331-336, 2007.

3. Shishido Y, Mitarai S, Otomo K, et al. Anti-tuberculosis drug susceptibility testing of Mycobacterium bovis BCG Tokyo strain. Int J Tuberc Lung Dis 11: 1334-1338, 2007.

4. Hesseling AC, Schaaf HS, Hanekom WA, et al. Danish Bacille Calmette-Guerin vaccine-induced disease in human immunodeficiency virus-infected children. Clin Infect Dis 37 1226-1233, 2003 (Erratum in: Clin Infect Dis 37: 1727, 2003).
5. Talbot EA, Perkins MD, Silva SF, Frothingham R. Disseminated bacilli Calmette-Guerin disease after vaccination: case report and review. Clin Infect Dis 24: 1139-1146, 1997.

6. Steg A, Leleu C, Debre B, Boccon-Gibod L, Sicard D. Systemic bacillus Calmette-Guerin infection, 'BCGitis' in patients treated by intravesical bacillus Calmette-Guerin therapy for bladder cancer. Eur Urol 16: 161-164, 1989.

7. Yamaga $\mathrm{H}$, Noda E, Yoshiyama $\mathrm{M}$, et al. BCG hinaisesshu no ichirei. Shouni Kansen Meneki 16: 163-166, 2004 (in Japanese).

(C) 2009 The Japanese Society of Internal Medicine http://www.naika.or.jp/imindex.html 